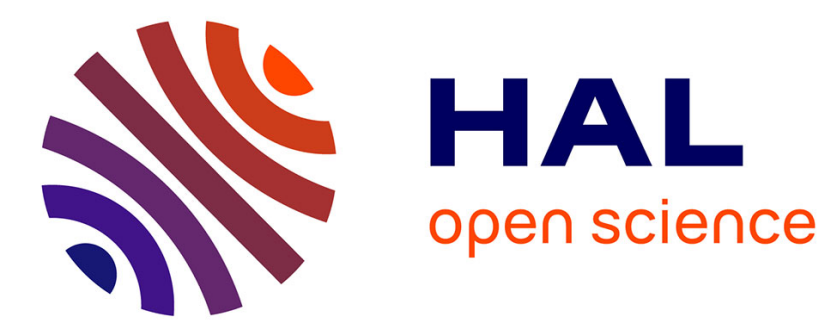

\title{
Laminar-turbulent boundary in plane Couette flow
}

Tobias M. Schneider, John F. Gibson, Maher Lagha, Filippo de Lillo, Bruno Eckhardt

\section{To cite this version:}

Tobias M. Schneider, John F. Gibson, Maher Lagha, Filippo de Lillo, Bruno Eckhardt. Laminarturbulent boundary in plane Couette flow. Physical Review E : Statistical, Nonlinear, and Soft Matter Physics, 2008, 78 (3), pp.037301. 10.1103/physreve.78.037301 . hal-01022806

\section{HAL Id: hal-01022806 \\ https://hal-polytechnique.archives-ouvertes.fr/hal-01022806}

Submitted on 21 Jul 2014

HAL is a multi-disciplinary open access archive for the deposit and dissemination of scientific research documents, whether they are published or not. The documents may come from teaching and research institutions in France or abroad, or from public or private research centers.
L'archive ouverte pluridisciplinaire HAL, est destinée au dépôt et à la diffusion de documents scientifiques de niveau recherche, publiés ou non, émanant des établissements d'enseignement et de recherche français ou étrangers, des laboratoires publics ou privés. 


\title{
Laminar-turbulent boundary in plane Couette flow
}

\author{
Tobias M. Schneider, ${ }^{1}$ John F. Gibson, ${ }^{2}$ Maher Lagha, ${ }^{3}$ \\ Filippo De Lillo, ${ }^{4,1}$ and Bruno Eckhardt ${ }^{1}$ \\ ${ }^{1}$ Fachbereich Physik, Philipps-Universität Marburg, D-35032 Marburg, Germany \\ ${ }^{2}$ Center for Nonlinear Science, School of Physics, Georgia Institute of Technology, Atlanta, Georgia 30332, USA \\ ${ }^{3}$ Laboratoire d'Hydrodynamique (LadHyX), CNRS-École Polytechnique, F-91128 Palaiseau, France \\ ${ }^{4}$ Università degli Studi di Torino, Dipartimento di Fisica Generale and Istituto Nazionale di Fisica Nucleare, sez. di Torino, \\ Via Giuria 1, I-10125, Torino, Italy
}

(Received 2 May 2008; published 8 September 2008)

\begin{abstract}
We apply the iterated edge-state tracking algorithm to study the boundary between laminar and turbulent dynamics in plane Couette flow at $\mathrm{Re}=400$. Perturbations that are not strong enough to become fully turbulent or weak enough to relaminarize tend toward a hyperbolic coherent structure in state space, termed the edge state, which seems to be unique up to obvious continuous shift symmetries. The results reported here show that in cases where a fixed point has only one unstable direction, such as for the lower-branch solution in plane Couette flow, the iterated edge tracking algorithm converges to this state. They also show that the choice of initial state is not critical and that essentially arbitrary initial conditions can be used to find the edge state.
\end{abstract}

DOI: 10.1103/PhysRevE.78.037301

PACS number(s): 47.10.Fg, 47.27.Cn, 47.27.ed

Plane Couette flow and pipe flow belong to the class of shear flows where turbulence occurs despite the persistent linear stability of the laminar profile [1]. Triggering turbulence then requires the crossing of two thresholds, one in Reynolds number and one in perturbation amplitude. Guidance on the minimum Reynolds number is offered by the appearance of exact coherent states: once they are present, an entanglement of their stable and unstable manifolds can provide the necessary state space elements for chaotic, turbulent dynamics [2-14]. Since the exact coherent states appear in saddle-node bifurcations, it is natural to associate the upper branch (characterized by a higher kinetic energy or a higher drag) with the turbulent dynamics and the lower branch with the threshold in perturbation amplitude [8,15-18]. In plane Couette flow this scenario seems to be borne out: at the point of bifurcation, at a Reynolds number of about 127.7, the upper branch state is stable and the lower one has only one unstable direction $[6,8,18]$. At slightly higher Reynolds numbers, the upper branch undergoes secondary bifurcations which could lead to the complex state space structure usually associated with turbulent dynamics. For the lower branch, on the other hand, there are no indications of further bifurcations. If it continues to have a single unstable direction only, its stable manifold can divide the state space such that initial conditions from one side decay more or less directly to the laminar profile, whereas those from the other side show some turbulent dynamics. Such a description of the transition along the lines of the phenomenology of saddle-node bifurcations has been advanced by Toh and Itano [15] for plane Poiseuille flow, by Wang et al. [18] and Viswanath [19,20] for plane Couette flow, and Kerswell and Tutty for pipe flow [21].

Empirically, one may study the boundary between laminar and turbulent dynamics by following the time evolution of flow fields and thereby assigning a lifetime-i.e., the time it takes for a particular initial condition to decay toward the laminar profile [22-25]. Increasing the amplitude of the perturbation one notes changes between regions with smooth variations in lifetimes (where trajectories decay rather di- rectly) and regions with huge fluctuations showing a sensitive dependence on initial conditions, since neighboring initial states can have vastly different lifetimes. A point on the border between laminar and chaotic regions is said to lie on the edge of chaos [24]. A trajectory starting from such a point will neither decay to the laminar state nor swing up to turbulence: it will move in intermediate regions between laminar and turbulent motion. All points visited will lie in the edge of chaos and can be identified by the above search using suitable initial conditions. What is interesting is that trajectories moving around in this edge of chaos are dynamically attracted to a subset of state space [13,24-28]. This subset is invariant under the flow and attracting for initial conditions within the edge of chaos: we call it the edge state. It is only a relative attractor, since it is unstable against perturbations that lead outside the edge of chaos.

The connection between this concept and the saddle-node approach described before is straightforward: if the boundary between laminar and turbulent regions is formed by the stable manifold of a saddle state, then the manifold coincides with the edge of chaos and the edge state is the saddle state itself. This is possible only if the saddle state has a single unstable direction. If further directions are unstable, the edge state will not be a fixed point, but a periodic orbit or a chaotic attractor: this seems to be the case in certain lowdimensional models and in pipe flow [24-26].

A trajectory in the edge can be bracketed by initial conditions on the laminar and the turbulent side-i.e., initial conditions which eventually decay or become turbulent. Technically, if we have an initial condition $\mathbf{u}$ that becomes turbulent, then we study, with $\mathbf{u}_{L}$ the laminar profile, the family of initial conditions

$$
\mathbf{u}_{\lambda}=\mathbf{u}_{L}+\lambda \mathbf{v},
$$

with $\mathbf{v}=\mathbf{u}-\mathbf{u}_{L}$. For $\lambda=1$, this is just the continuation of the previous trajectory. Reducing $\lambda$, the initial conditions move closer to the laminar profile and will not become turbulent. Therefore, one can find an interval of $\lambda$ values bounded at 

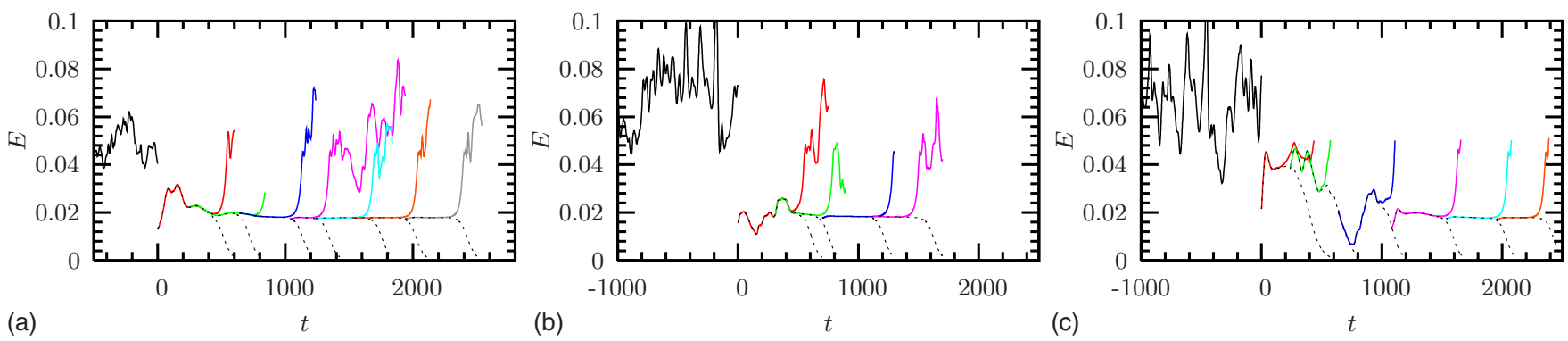

FIG. 1. (Color online) Time traces of the perturbation energy for three typical initial conditions, followed over several refinements in the edge tracking algorithm. The abscissa gives the time in units of $h / U_{0}$ and the ordinate the energy per volume. The solid lines are orbits that escape to the turbulent side and the dashed ones decay. Time zero corresponds to the time where the edge tracking algorithm starts.

one end by a trajectory that becomes turbulent and at the other by one that returns directly to the laminar profile. $\mathrm{Bi}$ secting in $\lambda$, we can focus on trajectories which reside for a substantial time interval without becoming turbulent or decaying toward the laminar profile. Since the two trajectories separate exponentially, they are followed for a finite time only and the bisection is repeated several times (see $[15,20,24,25]$ for descriptions of the method and Fig. 1 for an illustration for plane Couette flow). Different initial conditions can be expected to evolve toward the same edge state, unless there should be several coexisting ones.

The tracking of the dynamics in the edge without the requirement of an a priori knowledge of the hyperbolic structure offers several exciting possibilities: first of all, it allows one to study whether the dynamics in the edge is indeed attracted to some invariant flow structures. In principle, as the famous examples of Julia and Mandelbrot sets [29] and the findings for a simple model in [24] show, the dynamics in the edge could be persistent and periodic or even chaotic. Second, the convergence of the algorithm only requires the edge state to be an attracting set, without further assumptions about its nature (fixed point, periodic orbit, or chaotic attractor). Third, the rate of separation gives valuable dynamical information, as it limits the time interval over which the dynamics will be close to this edge state in numerical or experimental situations, unless further measures such as the edge tracking algorithm are implemented. The application to plane Couette flow given here demonstrates the versatility of the method and allows one to connect it to the ideas about lower-branch solutions advocated in [18].

On the numerical side, we solve the Navier-Stokes equations using a Fourier-Chebyshev- $\tau$ scheme [30,31]. The flow domain is set to be 2 units high, $2 \pi$ units wide, and $4 \pi$ units long and periodically continued in the spanwise and downstream directions. The coordinate system is chosen such that $x, y$, and $z$ correspond to the downstream, spanwise, and wall-normal directions, respectively. We use 64 Fourier modes in the $x$ and 32 in the $y$ direction and 25 Chebyshev modes in the $z$ direction. The equations for the wall-normal components of velocity $w$ and vorticity $\omega_{z}$ were solved, and the remaining components of velocity were computed by making use of the incompressibility condition $\boldsymbol{\nabla} \cdot \mathbf{u}=0$. The code is fully dealiased and was verified by reproducing the linear eigenvalue spectrum and the turbulent statistics. The Reynolds number is based on half the channel height and half the velocity difference between the plates. Units are cho- sen such that the velocity of the top plate is $U_{0}=1$, and time is measured in units of $h / U_{0}$, where $h$ is half the height of the channel. All calculations shown here are for a Reynolds number $\operatorname{Re}=U_{0} h / \nu=400$.

The time traces in Fig. 1 show that independent of the initial condition the edge tracking algorithm converges to one with constant energy. The initial and final states shown in Fig. 2 emphasize the very different topologies in the initial conditions, and the coincidence in the final states, modulo obvious translational shifts in the downstream or spanwise direction. Wang et al. [18] suggested that this state should coincide with a particular lower-branch solution. Their state is similar to, but as pointed out by Waleffe [33], not identical to the state found here. Introducing the wave numbers $\alpha$ and
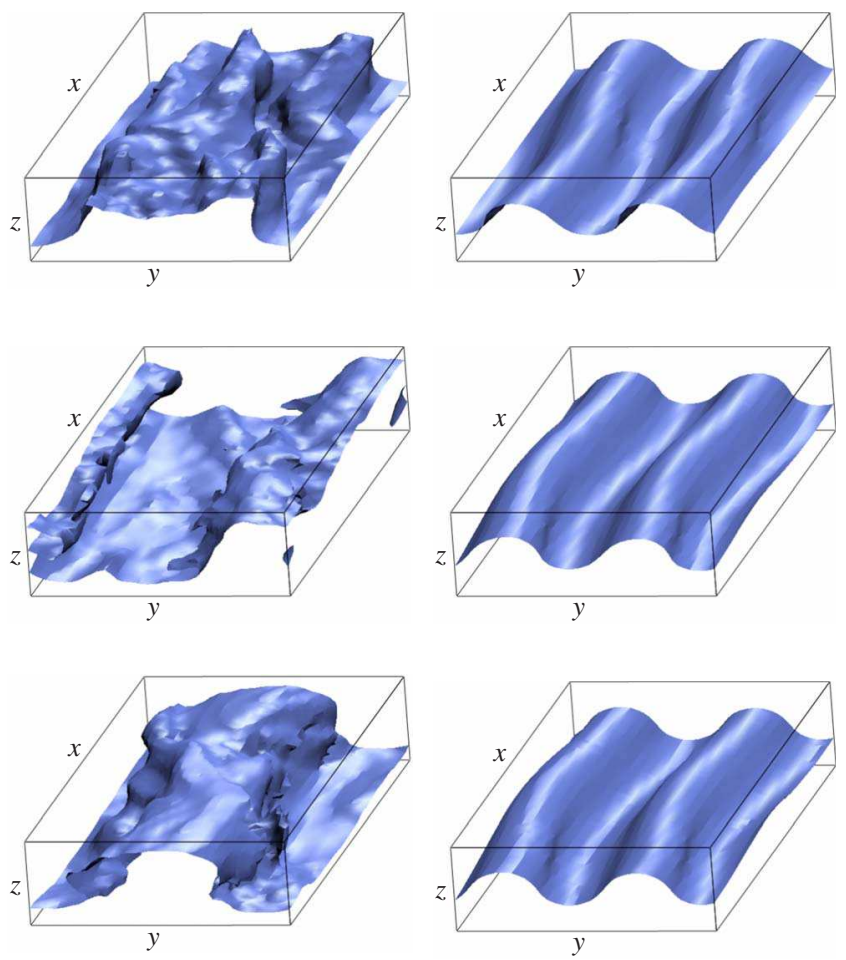

FIG. 2. (Color online) Isosurfaces for $v_{x}=0$ for the three trajectories shown in Fig. 1. The left column shows the initial state taken from the turbulent run, and the right column the final state obtained after iteration times 1568, 1250, and 2100. Note the reduced fluctuations. The final states coincide up to a shift in the downstream direction. 


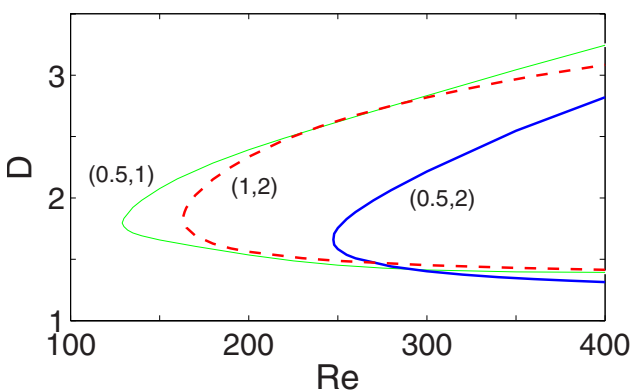

FIG. 3. (Color online) Bifurcation diagram for three pairs of lower- and upper-branch equilibrium solutions, with $(\alpha, \gamma)$ $=(0.5,1),(0.5,2)$, and $(1,2)$. The drag $D$ is the wall drag normalized by the drag of laminar flow at the same Reynolds number. For $\operatorname{Re}=400$, the $(0.5,2)$ state has the lowest drag.

$\gamma$ for the structures, so that the periods in the downstream and spanwise directions are $2 \pi / \alpha$ and $2 \pi / \gamma$, the state found by the edge tracking algorithm is $(\alpha, \gamma)=(0.5,2)$, whereas [18] suggested a state with $(\alpha, \gamma)=(0.5,1)$. In order to explain this difference, we show in Fig. 3 the bifurcation diagram for three states that fit into the periodic domain. This diagram suggests that the $(0.5,2)$ state could be on the edge for $\mathrm{Re}=400$. However, the drag corresponds to a specific projection of the high-dimensional state space only. Thus, in view of the discussion about the number of unstable directions, we need to verify that this state does not have more than one unstable direction.

Figure 4 shows the $(\alpha, \gamma)=(0.5,1),(0.5,2)$, and $(1,2)$ states and their eigenvalue spectra. The eigenvalues were computed with Arnoldi iteration on a $N_{x} \times N_{y} \times N_{z}=32 \times 49$ $\times 64$ grid. Recomputing on a $24 \times 35 \times 48$ grid confirmed their accuracy to three digits. Note that $N_{y} / L_{y}=4 N_{x} / L_{x}$, making the spatial resolution 4 times finer in $y$ than in $x$. This is best for the problem at hand because the Fourier spectrum is far from isotropic. All states have two neutral eigenvalues (not shown) from the neutral shifts in the periodic directions downstream and spanwise. The number of unstable direc- tions (where complex eigenvalues come in pairs and count as two directions) is 4,1 , and 5 for the states $(\alpha, \gamma)=(0.5,1)$, $(0.5,2)$, and $(1,2)$, respectively. Thus only the $(0.5,2)$ state has only one unstable direction, consistent with the observed convergence of the edge-state tracking algorithm.

The initial conditions used here are typical in the sense that they are taken from a turbulent velocity field. Previous studies have focused on initial conditions with certain properties, such as modulated downstream vortices or oblique waves. It seems that all of them can be used to track the edge of chaos and that all converge to the same invariant edge state, modulo translational symmetries. In principle, there could be more than one local attractor embedded in the edge as in highly symmetric constrained pipe flow [24,27,28]. However, since all edge tracking runs converged to the same flow field, we conclude that the edge state is either unique or that a possible second local attractor is located in a dynamically less relevant part of the edge.

The observation that the application of the refined edge tracking method to plane Couette flow converges to the lower branch solution, independent of initial conditions, also has implications for the observations on pipe flow: the fact that no steady or traveling wave invariant object is found even in a periodically continued pipe segment of fivediameter length, which corresponds to pipe flow restricted to a discrete translational symmetry, suggests very strongly that the invariant object is not simple, in the sense that it has more than one unstable direction, resulting in a chaotic edge state. Obviously, if it is chaotic, there will be simpler objects embedded and the coherent traveling waves identified by Pringle and Kerswell [32] could be first examples of such structures.

To summarize, the refined edge tracking algorithm applied here to plane Couette flow in a parameter range where the lower-branch solutions havd a stable manifold of codimension 1 confirms the expectations based on the saddlenode bifurcation scenario, but in addition it helps to identify which state among several possible ones is indeed the invariant object in the edge-i.e., the edge state. This confirms the
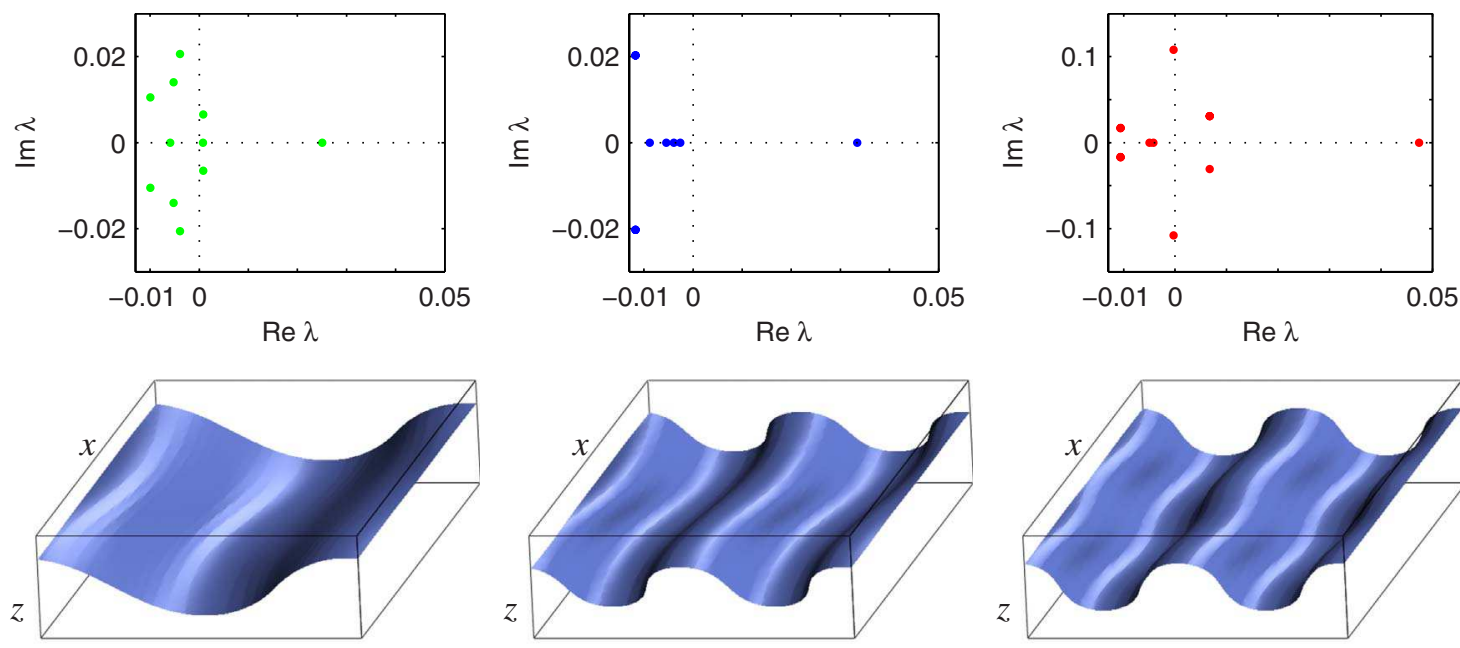

$y$

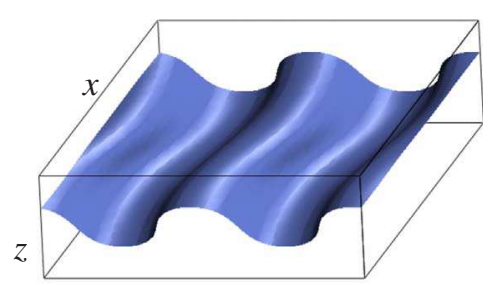

$y$

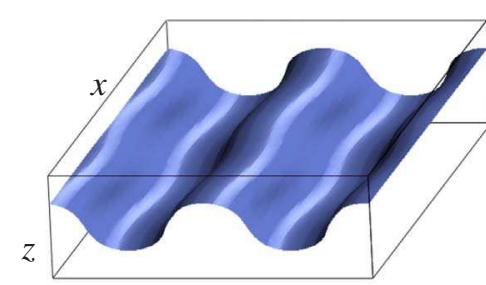

$y$

FIG. 4. (Color online) Eigenvalue spectra (top row) and velocity fields (bottom row) for the states (from left to right) $(\alpha, \gamma)=(0.5,1)$, $(0.5,2)$, and $(1,2)$ at $\operatorname{Re}=400$. 
robustness of the algorithm and demonstrates that it is capable of identifying the edge state as the relative attractor in the edge of chaos. Specifically, if the edge state has only one unstable direction, it manages to find the corresponding invariant coherent state, since only the energy content is controlled. Conversely, if it does not converge to a simple invariant object, this must be due to the presence of more than one unstable direction. Of course, if the edge state is dynamically nontrivial and chaotic (i.e., neither a steady state nor a traveling wave), then it will contain simpler periodic or relatively periodic structures, but they will have more than one unstable direction so that their stable manifold has codimension higher than 1 and cannot divide the state space. This seems to be the case for all coherent structures observed thus far in pipe flow. The versatility of the method immediately suggests applications to a variety of other shear flows where laminar and turbulent dynamics coexist.

We thank Fabian Waleffe for his helpful comments and for his identification of the differences in the edge states. This work was supported in part by the Deutsche Forschungsgemeinschaft.
[1] S. Grossmann, Rev. Mod. Phys. 72, 603 (2000).

[2] M. Nagata, J. Fluid Mech. 217, 519 (1990).

[3] M. Nagata, Phys. Rev. E 55, 2023 (1997).

[4] M. Nagata, J. Fluid Mech. 307, 231 (1996).

[5] M. Nagata, J. Fluid Mech. 358, 357 (1998).

[6] R. Clever and F. H. Busse, J. Fluid Mech. 344, 137 (1997).

[7] F. Waleffe, Phys. Rev. Lett. 81, 4140 (1998).

[8] F. Waleffe, Phys. Fluids 15, 1517 (2003).

[9] H. Faisst and B. Eckhardt, Phys. Rev. Lett. 91, 224502 (2003).

[10] H. Wedin and R. R. Kerswell, J. Fluid Mech. 508, 333 (2004).

[11] G. Kawahara and S. Kida, J. Fluid Mech. 449, 291 (2001).

[12] R. R. Kerswell, Nonlinearity 18, R17 (2005).

[13] B. Eckhardt, T. M. Schneider, B. Hof, and J. Westerweel, Annu. Rev. Fluid Mech. 39, 447 (2007).

[14] B. Eckhardt, Nonlinearity 21, T1 (2008).

[15] S. Toh and T. Itano, e-print arXiv:physics/9905012.

[16] T. Itano and S. Toh, J. Phys. Soc. Jpn. 70, 703 (2001).

[17] F. Waleffe, J. Fluid Mech. 435, 93 (2001).

[18] J. Wang, J. Gibson, and F. Waleffe, Phys. Rev. Lett. 98, 204501 (2007).

[19] D. Viswanath, e-print arXiv:physics/0701337.

[20] D. Viswanath, J. Fluid Mech. 580, 339 (2007).
[21] R. R. Kerswell and O. R. Tutty, J. Fluid Mech. 584, 69 (2007).

[22] A. Schmiegel and B. Eckhardt, Phys. Rev. Lett. 79, 5250 (1997).

[23] H. Faisst and B. Eckhardt, J. Fluid Mech. 504, 343 (2004).

[24] J. D. Skufca, J. A. Yorke, and B. Eckhardt, Phys. Rev. Lett. 96, 174101 (2006).

[25] T. M. Schneider, B. Eckhardt, and J. A. Yorke, Phys. Rev. Lett. 99, 034502 (2007).

[26] T. M. Schneider and B. Eckhardt, Chaos 16, 041103 (2006).

[27] T. M. Schneider and B. Eckhardt, Bull. Am. Phys. Soc. 52 (17), 30 (2007).

[28] Y. Duguet, A. P. Willis, and R. R. Kerswell, e-print arXiv:0711.2175.

[29] H. Peitgen and P. Richter, The Beauty of Fractals: Images of Complex Dynamical Systems (Springer, Berlin, 2000).

[30] C. Canuto, M. Y. Hussaini, A. Quarteroni, and T. A. Zang, Spectral Methods in Fluid Dynamics (Springer-Verlag, Berlin, 1988).

[31] F. De Lillo and B. Eckhardt, Phys. Rev. E 76, 016301 (2007).

[32] C. C. T. Pringle and R. R. Kerswell, Phys. Rev. Lett. 99, 074502 (2007).

[33] F. Waleffe (private communication). 